\title{
A Computational Technique for Evaluating $L(1, \chi)$ and the Class Number of a Real Quadratic Field
}

By H. C. Williams and J. Broere

\begin{abstract}
A description is given of a method for estimating $L(1, \chi)$ to sufficient accuracy to determine the class number of a real quadratic field. This algorithm was implemented on an IBM/370-158 computer and the class number, regulator, and value of $L(1, x)$ were obtained for each real quadratic field $Q(\sqrt{D})(D=$ $2,3, \ldots, 149999)$. Several tables, summarizing various results of these computations, are also presented.
\end{abstract}

1. Introduction. Recently Hendy [2] has calculated on a Burroughs B6700 computer the class numbers and number of genera for all the real quadratic fields $Q(\sqrt{D})$, with $10^{3} \leqslant D \leqslant 10^{5}$ and $D$ squarefree. The method he used to do this is a modification of Ince's [3] technique of counting periods. In this paper we describe an entirely different computational procedure for determining the class number of $Q(\sqrt{D})$ via its Dirichlet function $L(1, \chi)$. This algorithm was implemented on an IBM/370-158 computer and used to determine all the class numbers in the range $2 \leqslant$ $D \leqslant 1.5 \times 10^{5}$.

Our method is based upon the formula

$$
h=\sqrt{\Delta} L(1, \chi) / 2 R,
$$

where $h$ is the class number, $R$ is the regulator and $\Delta$ is the discriminant of $Q(\sqrt{D})$. The Dirichlet series $L(s, \chi)=\sum_{n=1}^{\infty} \chi(n) n^{-s}$ has $\chi(n)=(\Delta \mid n)$ (the Kronecker Symbol). It should be remarked here that

$$
\Delta= \begin{cases}D, & D \equiv 1(\bmod 4), \\ 4 D, & D \equiv 2,3(\bmod 4),\end{cases}
$$

and $R=\log \epsilon$, where $\epsilon(>1)$ is the fundamental unit of $Q(\sqrt{D})$.

In the next section we describe a means of evaluating $R$ and in the third section a method of estimating $L(1, \chi)$ to sufficient accuracy to determine the integer $h$. Finally, we give some results of our computations in Section 4.

2. Evaluation of the Regulator. The regulator can be evaluated by using the continued fraction algorithm. Put $Q_{0}=1, P_{0}=0, q_{0}=[\sqrt{D}]^{*}, A_{-1}=B_{-2}=1$, $A_{-2}=B_{-1}=0$, and define

$$
\begin{gathered}
P_{n+1}=q_{n} Q_{n}-P_{n}, \quad Q_{n+1}=\left(D-P_{n+1}^{2}\right) / Q_{n}, \quad q_{n+1}=\left[\left(P_{n+1}+\sqrt{D}\right) / Q_{n+1}\right], \\
A_{n+1}=q_{n+1} A_{n}+A_{n-1}, \quad B_{n+1}=q_{n+1} B_{n}+B_{n-1} .
\end{gathered}
$$

Received December 12, 1975; revised January 23, 1976.

AMS (MOS) subject classifications (1970). Primary 12A70, 12 A25, 12-04, 12 A50.

${ }^{*}$ We use the usual notation $[\alpha]$ to indicate the greatest integer $\leqslant \alpha$. 
Let $r$ be the least nonnegative integer such that $Q_{r+1}=4$, and let $s$ be the least nonnegative integer such that $Q_{s+1}=1(s+1$ is the period length of the continued fraction for $\sqrt{D}$ ). It is well known that such a value of $s$ always exists; and that if $r$ exists, then $r<s / 2$. With these definitions of $r$ and $s$ and $D \neq 5$ we have

$$
\epsilon= \begin{cases}\left(A_{r}+\sqrt{D} B_{r}\right) / 2 & \text { if } r \text { exists, } \\ A_{s}+\sqrt{D} B_{s} & \text { otherwise. }\end{cases}
$$

A faster and more convenient way to calculate the regulator, however, can be obtained by first using the following modification of the continued fraction algorithm to evaluate the $P$ 's and $Q$ 's. We put $Q_{-1}=D, R_{0}=0$ and use the formulas

$$
\begin{gathered}
P_{n+1}=[\sqrt{D}]-R_{n}, \quad Q_{n+1}=Q_{n-1}+q_{n}\left(P_{n}-P_{n+1}\right), \\
q_{n+1}=\left[\left(P_{n+1}+[\sqrt{D}]\right) / Q_{n+1}\right] \\
R_{n+1}=\text { remainder on dividing } P_{n+1}+[\sqrt{D}] \text { by } Q_{n+1} .
\end{gathered}
$$

We can then avoid calculating the $A$ 's and $B$ 's by using a multiplicative formulation (see Smith [5]) wherein

$$
(-1)^{n+1}\left(A_{n}-\sqrt{D} B_{n}\right)=\left(\prod_{i=1}^{n+1}\left(P_{i}+\sqrt{D}\right) / Q_{i}\right)^{-1} \text {. }
$$

Since $A_{r}^{2}-D B_{r}^{2}=4(-1)^{r+1}$, we get

$$
\left(A_{r}+\sqrt{D} B_{r}\right) / 2=2 \prod_{i=1}^{r+1}\left(P_{i}+\sqrt{D}\right) / Q_{i}
$$

Now during the development of the $P$ 's and $Q$ 's there must be an integer $k$ such that $Q_{k}=Q_{k+1}$ or an integer $j$ such that $P_{j}=P_{j+1}$. If $k$ exists, then $s+1=$ $2 k+1$; if $j$ exists, $s+1=2 j$; also, $r+1 \leqslant k, j$. Using these facts to determine $k$ or $j$, we can cut the calculation of $A_{s}+\sqrt{D} B_{s}$ in half by using the symmetry properties of the $P$ 's and $Q$ 's and the fact that $A_{s}^{2}-D B_{s}^{2}=(-1)^{s+1}$. We have

$$
A_{s}+\sqrt{D} B_{s}=\left(P_{k+1}+\sqrt{D}\right)\left[\prod_{i=1}^{k}\left(P_{i}+\sqrt{D}\right) / Q_{i}\right]^{2} \quad \text { when } s+1=2 k+1
$$

or

$$
A_{s}+\sqrt{D} B_{s}=Q_{j}\left[\prod_{i=1}^{j}\left(P_{j}+\sqrt{D}\right) / Q_{i}\right]^{2} \quad \text { when } s+1=2 j
$$

hence,

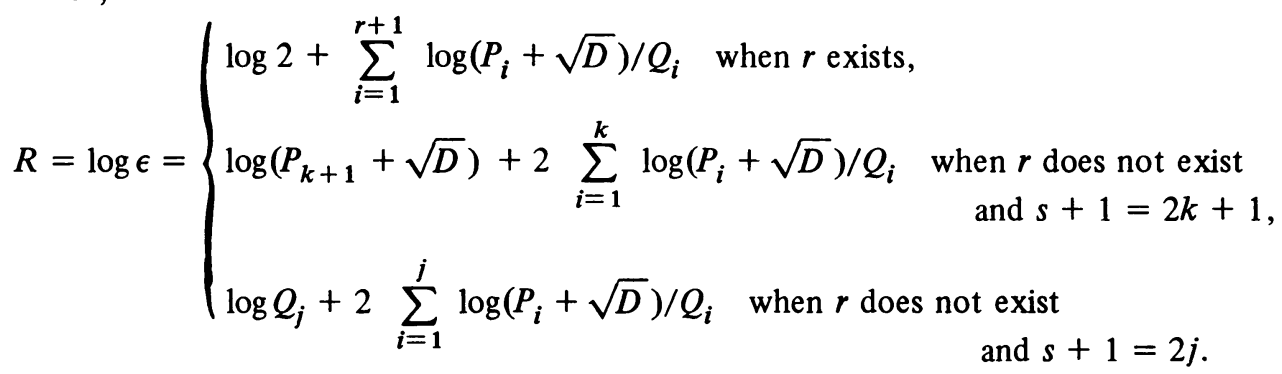


3. Estimation of $L(1, \chi)$. In order to estimate $L(1, \chi)$ we make use of a device used by Barrucand [1]. We first note that $L(s, \chi)$ satisfies the functional equation

$$
L(1-s, \chi)=A^{-s+1 / 2} \frac{\Gamma(s / 2)}{\Gamma((1-s) / 2)} L(s, \chi),
$$

where $A=\pi / \Delta$. Putting $A=\alpha \beta$, we get

$$
\alpha^{-s+1 / 2} \Gamma(s / 2) L(s, \chi)=\beta^{s-1 / 2} \Gamma((1-s) / 2) L(1-s, \chi) .
$$

If we let

$$
B(x)=\sum_{n=1}^{\infty} \chi(n) e^{-n^{2} x}
$$

and use the method of [1], we obtain the functional equation

$$
\sqrt{\alpha} B\left(\alpha^{2} x\right)=\sqrt{\beta} x^{-1 / 2} B\left(\beta^{2} / x\right) .
$$

Hence,

$$
\begin{aligned}
& \alpha^{-s} L(s, \chi) \Gamma(s / 2)=\int_{0}^{\infty} B\left(\alpha^{2} x\right) x^{s / 2-1} d x \\
& \quad=\int_{1}^{\infty} B\left(\alpha^{2} x\right) x^{s / 2-1} d x+\sqrt{\beta / \alpha} \int_{0}^{1} B\left(\beta^{2} / x\right) x^{(s-3) / 2} d x \\
& \quad=\sum_{n=1}^{\infty} \chi(n) \int_{1}^{\infty} e^{-\alpha^{2} n^{2} x} x^{s / 2-1} d x+\sqrt{\beta / \alpha} \sum_{n=1}^{\infty} \chi(n) \int_{0}^{1} x^{(s-3) / 2} e^{-\beta^{2} n^{2} / x} d x .
\end{aligned}
$$

If we put $\alpha=\beta=\sqrt{A}, s=1$,

we get

$$
E(x)=\int_{x}^{\infty} e^{-t / t} d t, \quad \operatorname{erfc}(x)=\frac{2}{\sqrt{\pi}} \int_{x}^{\infty} e^{-t^{2}} d t
$$

$$
L(1, \chi)=\frac{1}{\sqrt{\Delta}} \sum_{n=1}^{\infty} \chi(n) E\left(A n^{2}\right)+\sum_{n=1}^{\infty}(\chi(n) / n) \operatorname{erfc}(n \sqrt{A}) .
$$

Let

$$
\begin{aligned}
& C(m)=\frac{1}{\sqrt{\Delta}} \sum_{n=1}^{m} \chi(n) E\left(A n^{2}\right)+\sum_{n=1}^{m}(\chi(n) / n) \operatorname{erfc}(n \sqrt{A}), \\
& T(m)=\frac{1}{\sqrt{\Delta}} \sum_{n=m+1}^{\infty} \chi(n) E\left(A n^{2}\right)+\sum_{n=m+1}^{\infty}(\chi(n) / n) \operatorname{erfc}(n \sqrt{A}) .
\end{aligned}
$$

Since we wish to approximate $L(1, \chi)$ by the partial sum $C(m)$, we want to be able to determine $m$ such that the remainder $T(m)$ is small enough that the integer $h$ can be determined unequivocally. Now if $x>0$,

$$
0<\operatorname{erfc}(x)<\frac{1}{x \sqrt{\pi}} e^{-x^{2}} \text { and } 0<E(x)<e^{-x} / x
$$

thus, since $|\chi(n)| \leqslant 1$, we get

$$
|T(m)|<\frac{2 \sqrt{\Delta}}{\pi} \sum_{n=m+1}^{\infty} e^{-A n^{2}} / n^{2}<\frac{2 \sqrt{\Delta}}{\pi} \int_{m}^{\infty} e^{-A t^{2}} / t^{2} d t<\frac{\Delta^{3 / 2}}{\pi^{2}} e^{-A m^{2}} / m^{3} .
$$

If we let $h^{*}=\sqrt{\Delta} C(m) / 2 R$, then 


$$
\left|h-h^{*}\right|<\frac{\sqrt{\Delta}}{2 R}|T(m)|<\frac{A^{2}}{2 R m^{3}} e^{-A m^{2}} .
$$

Since $h$ is an integer and $2^{t-\lambda} \mid h$, where $t$ is the number of distinct prime factors of $\Delta$ and

$$
\lambda= \begin{cases}1 & \text { if all prime divisors of } D \text { are congruent to } 1,2(\bmod 4), \\ 2 & \text { otherwise, }\end{cases}
$$

we must find $m$ such that $A^{2} e^{-A m^{2}} / m^{3}<2^{t-\lambda} R$. When this is done, $h$ is the unique integer, which is divisible by $2^{t-\lambda}$, in the interval $\left[h^{*}-2^{t-\lambda-1}, h^{*}+2^{t-\lambda-1}\right]$.

If we put $c=\sqrt{A} m$ and $l=\log \left(\sqrt{A} / 2^{t-\lambda} R\right)$, we must find $c$ such that $c^{2}+$ $3 \log c>l$. If $l>1$ and $\Delta<10^{7}$, then $1<c<2$; thus, $c=1+\epsilon, \epsilon<1$ and $\log c>$ $\epsilon-\epsilon^{2} / 2$. If $l<1$, then $1 / 2<c<1$ and $c=1-\epsilon>1-2 \epsilon^{2}>e^{-2 \epsilon}$; hence, $\log c>$ $2(c-1)$. If we use these results, we see that we may put $m=[c \sqrt{\Delta} / \sqrt{\pi}]+1$, where

$$
c= \begin{cases}6-\sqrt{27-2 l}, & l>1 \\ \sqrt{15+l}-3, & l<1\end{cases}
$$

For $\Delta<6 \times 10^{5}$ it is rarely necessary to go beyond 500 terms in the series $C(m)$ in order to evaluate $h$.

4. Results of the Computations. The program which evaluated $C(m)$ and then $h$, was written, using double precision, in FORTRAN. A special subroutine to evaluate $E(x)$ in double precision was written in assembler language, and the FORTRAN function DERFC was used to evaluate $\operatorname{erfc}(x)$. In about seven hours of CPU time the computer calculated the class numbers of $Q(\sqrt{D})$ for all squarefree $D$ such that $1<$ $D \leqslant 1.5 \times 10^{5}$. These class numbers for $10^{3} \leqslant D \leqslant 10^{5}$ probably agree entirely with those in Hendy's table since the number of $D$, between these limits, having a given $h$ agree in the two tables. Once $h$ had been calculated for $Q(\sqrt{D})$, the value of $L(1, \chi)$ was calculated more precisely by using $L(1, \chi)=2 R h / \sqrt{\Delta}$.

A large table, listing for each of the $91189 Q(\sqrt{D})$, the regulator, the class number, and the value of $L(1, \chi)$ has been deposited in the UMT file. In this section we present some excerpts from that table. In Table 1 we give each value of $h$ which occurs in the large table, the frequency $f(h)$ with which this $h$ occurs, and the least value of $D$ such that $h$ is the class number for $Q(\sqrt{D})$.

Denote by $R(d)$ the regulator of $Q(\sqrt{d})$ and by $L\left(1, \chi_{\delta}\right)$ the value of $L(1, \chi)$ when $\chi(n)=(\delta \mid n)$. In Table 2 we give those values of $D$ and $R(D)$ where $R(D)$ attains a new maximum:

$$
R(D)>R(d) \quad \text { for all } 2 \leqslant d<D .
$$

In his examination of Littlewood's bounds on $L(1, \chi)$, Shanks [4] considered the function

$$
L_{-\delta}(1)=\sum_{m=1}^{\infty}(4 \delta \mid m) m^{-1}
$$


TABLE 1

\begin{tabular}{|c|c|c|c|c|c|}
\hline $\mathrm{h}$ & $f(h)$ & least $\mathrm{D}$ & in & $f^{\prime}(i)$ & least D \\
\hline 1 & 20574 & 2 & 2 & 26427 & 10 \\
\hline 3 & 2677 & 79 & 4 & 18573 & 82 \\
\hline 5 & 943 & 401 & 6 & 3453 & 235 \\
\hline 7 & 462 & 577 & 8 & 6898 & 226 \\
\hline 9 & 311 & 1129 & 10 & 1237 & 1111 \\
\hline 11 & 176 & 1297 & 12 & 2434 & 730 \\
\hline 13 & 124 & 4759 & 14 & 563 & $153^{\prime} t$ \\
\hline 15 & 115 & 9871 & 16 & 1970 & 2305 \\
\hline 17 & 62 & 7054 & 18 & 385 & 4954 \\
\hline 19 & 48 & 15409 & 20 & 788 & 3601 \\
\hline 21 & 43 & 7057 & 22 & 163 & 4762 \\
\hline 23 & 20 & 23593 & 24 & 838 & 9634 \\
\hline 25 & 30 & 24859 & 26 & 110 & $1332 . \mathrm{L}$ \\
\hline 27 & 20 & 8761 & 28 & 324 & 5626 \\
\hline 29 & 16 & 49281 & 30 & 113 & 11665 \\
\hline 31 & 4 & 97753 & 32 & 397 & 15130 \\
\hline 33 & 11 & 55339 & 34 & 47 & 19882 \\
\hline 35 & 8 & 25601 & 36 & 165 & 18226 \\
\hline 37 & 7 & 24337 & 38 & 33 & 19834 \\
\hline 39 & 6 & 41614 & 40 & 179 & 16899 \\
\hline 41 & 1 & 55966 & 42 & 30 & 47959 \\
\hline 43 & 3 & 14401 & 44 & 82 & 11026 \\
\hline 45 & 7 & 32401 & 46 & 14 & 49321 \\
\hline 47 & 1 & 78401 & 48 & 92 & 21610 \\
\hline 49 & 1 & 70969 & 50 & 8 & 54769 \\
\hline 51 & 1 & 69697 & 52 & 28 & 23410 \\
\hline 53 & 1 & 69694 & 54 & 8 & 49834 \\
\hline 55 & 1 & 106537 & 56 & 38 & 39999 \\
\hline 57 & 2 & 41617 & 58 & 7 & 27226 \\
\hline 60 & 18 & 78745 & 61 & 1 & 126499 \\
\hline 62 & 3 & 68179 & 63 & 1 & 57601 \\
\hline 64 & 23 & 71290 & 66 & 3 & 87271 \\
\hline 68 & 12 & 53362 & 70 & 5 & 56011 \\
\hline 72 & 11 & 45511 & 74 & 1 & 38026 \\
\hline 76 & 7 & 93618 & 78 & 1 & 136159 \\
\hline 80 & 3 & 94546 & 84 & 3 & 77779 \\
\hline 86 & 2 & 1.20926 & 87 & 2 & 90001 \\
\hline 88 & 3 & 56170 & 94 & 2 & 99226 \\
\hline 96 & 4 & 50626 & 100 & 2 & 131770 \\
\hline 108 & 1 & 140626 & 110 & 1 & 125434 \\
\hline 116 & 1 & 116554 & & & \\
\hline
\end{tabular}

Here we have

$$
L_{-\delta}(1)= \begin{cases}1 / 2 L\left(1, \chi_{\delta}\right), & \delta \equiv 1(\bmod 8), \\ 3 L\left(1, \chi_{\delta}\right) / 2, & \delta \equiv 5(\bmod 8), \\ L\left(1, \chi_{\delta}\right), & \text { otherwise }\end{cases}
$$

In Table 3 we give the values of $D, L_{-D}(1)$ (also the "Upper Littlewood Index" ULI [4]) such that

$$
L_{-D}(1)>L_{-\delta}(1) \text { for all } 2 \leqslant \delta<D \text {. }
$$

This gives us an extension of Shanks' table of Hichamps [4, Table 6]. 
TABLE 2 $R(D)$

\begin{tabular}{|c|c|c|c|}
\hline D & $R(D)$ & D & $R(D)$ \\
\hline 2 & 0.88137359 & 9619 & 239.95274415 \\
\hline 3 & 1.31605790 & $\therefore 11: 399$ & 255.84851576 \\
\hline 6 & $2.2924316 ?$ & 10651 & 270.87206891 \\
\hline 7 & $2.76865 \div 38$ & 12919 & 283.24482085 \\
\hline 11 & 2.99322285 & 13126 & 298.64260332 \\
\hline 14 & 3.40008441 & 15031 & 303.73613093 \\
\hline 19 & 5.82893697 & 16699 & 306.31406366 \\
\hline 22 & 5.97634447 & 16879 & 318.45171155 \\
\hline 31 & 8.01961269 & 17494 & 335.65693960 \\
\hline 43 & 8.84850928 & 17614 & 336.73823980 \\
\hline 46 & 10.79281810 & 18379 & 367.19773204 \\
\hline 67 & 11.48949306 & 21319 & 392.01026227 \\
\hline 94 & 15.27100210 & 23566 & 397.85610155 \\
\hline 109 & 16.69360526 & 23599 & 400.38847076 \\
\hline 139 & 18.85975147 & 25939 & 415.06367196 \\
\hline 151 & 21.96346336 & 26959 & 423.40328648 \\
\hline 199 & 24.20550214 & 27934 & 433.05457646 \\
\hline 211 & 27.04530804 & 28414 & 447.08037149 \\
\hline 214 & 27.36084155 & 31606 & 456.89547593 \\
\hline 331 & 36.25638320 & 32839 & 458.83193172 \\
\hline 379 & 37.79233938 & 32971 & 502.24984001 \\
\hline 526 & 46.57116319 & 34654 & 508.58627196 \\
\hline 571 & 47.33886269 & 38119 & 525.24115870 \\
\hline 631 & 52.93846995 & 42046 & 532.11987985 \\
\hline 739 & 53.63256141 & 42571 & 538.69114448 \\
\hline 751 & 57.94214806 & 43726 & 550.86782494 \\
\hline 886 & 58.00204637 & 46006 & 585.68371690 \\
\hline 919 & 64.36292549 & 48799 & 619.57038850 \\
\hline 991 & 68.80184250 & 53299 & 645.02269054 \\
\hline 1291 & 69.42731847 & 55819 & 646.58791328 \\
\hline 1366 & 77.50745983 & 56611 & 647.63115251 \\
\hline 1699 & 77.68763324 & 58774 & 649.84304001 \\
\hline 1726 & 91.48344937 & 60811 & 653.01948610 \\
\hline 1999 & 91.88716165 & 61051 & 700.82741506 \\
\hline 2011 & 100.53300453 & 67846 & 725.32530214 \\
\hline 2311 & 110.30316856 & 72934 & 737.97224426 \\
\hline 2326 & 111.22886783 & 76651 & 754.29325713 \\
\hline 2566 & 114.05602902 & 78094 & 795.25078126 \\
\hline 2671 & 119.59493590 & 78439 & 813.56346791 \\
\hline 3019 & 127.49681351 & 82471 & 817.86184239 \\
\hline 3259 & 132.12648968 & 84991 & 822.16136682 \\
\hline 3691 & 137.398241 .37 & 85999 & 826.11841497 \\
\hline 3931 & 147.25726673 & 87151 & 841.01248095 \\
\hline 4174 & 153.01734303 & 90931 & 867.19521570 \\
\hline 4846 & 162.46487523 & 98011 & 867.76246000 \\
\hline 4951 & 166.65898164 & 100291 & 879.44151133 \\
\hline 5119 & 172.50838882 & 102859 & 894.92275682 \\
\hline 6211 & 174.49073086 & 104311 & 907.83373877 \\
\hline 6379 & 175.31521179 & 106591 & 922.69477242 \\
\hline 6406 & 188.37917309 & 111094 & 971.04549162 \\
\hline 6451 & 196.13099779 & 122719 & 982.46753897 \\
\hline 7606 & 215.68131176 & 132694 & 985.17025244 \\
\hline 8254 & 221.19253648 & 133519 & 1029.90983807 \\
\hline 8779 & 231.75791826 & 139591 & 1063.78482684 \\
\hline
\end{tabular}


TABLE 3

\begin{tabular}{r|c|c|r|c|c}
$\mathrm{D}$ & \multicolumn{1}{c}{$\mathrm{L}_{-\mathrm{D}}(1)$} & ULI & $\mathrm{D}$ & $\mathrm{L}_{-D}(1)$ & ULI \\
\hline 2 & 0.62322524 & 0.4780 & 2146 & 2.31103546 & 0.5888 \\
3 & 0.76034600 & 0.4690 & 2479 & 2.31375725 & 0.5853 \\
6 & 0.93588131 & 0.4544 & 2599 & 2.34972306 & 0.5931 \\
7 & 1.04645488 & 0.4881 & 3826 & 2.45090149 & 0.6074 \\
10 & 1.15008652 & 0.4947 & 5014 & 2.45196600 & 0.6003 \\
19 & 1.33724985 & 0.5122 & 5251 & 2.47339296 & 0.6044 \\
31 & 1.44036496 & 0.5142 & 7459 & 2.53759015 & 0.6108 \\
34 & 1.45715183 & 0.5140 & 8551 & 2.54931771 & 0.6102 \\
46 & 1.59131421 & 0.5410 & 9454 & 2.57982512 & 0.6150 \\
79 & 1.71299181 & 0.5495 & 10651 & 2.62463570 & 0.6227 \\
106 & 1.74607012 & 0.5446 & 13666 & 2.67064271 & 0.6275 \\
151 & 1.78736130 & 0.5404 & 18379 & 2.70856368 & 0.6293 \\
211 & 1.86187579 & 0.5479 & 22234 & 2.76477214 & 0.6380 \\
214 & 1.91136378 & 0.5619 & 32971 & 2.76601001 & 0.6295 \\
274 & 1.91926263 & 0.5538 & 39274 & 2.77587028 & 0.6279 \\
331 & 1.99283105 & 0.5673 & 45046 & 2.79115252 & 0.6285 \\
394 & 2.06430094 & 0.5806 & 48799 & 2.80469210 & 0.6299 \\
631 & 2.10744721 & 0.5748 & 61051 & 2.83638181 & 0.6324 \\
751 & 2.11433901 & 0.5706 & 62386 & 2.34175734 & 0.6332 \\
919 & 2.12313701 & 0.5662 & 74299 & 2.85281091 & 0.6321 \\
991 & 2.18556256 & 0.5803 & 78439 & 2.90486139 & 0.6425 \\
1054 & 2.24501069 & 0.5940 & 84319 & 2.91010275 & 0.6423 \\
1486 & 2.26360155 & 0.5878 & 111094 & 2.91336081 & 0.6376 \\
1654 & 2.27963311 & 0.5886 & 127906 & 2.93902278 & 0.6406 \\
\hline
\end{tabular}

No further information was found to add to Shanks' table of Lochamps [4, Table 4]. There is no value of $D$ in the interval $2 \times 10^{3}<D<1.5 \times 10^{5}$ for which

$$
L_{-D}(1)<L_{-398}(1)=0.33494376 .
$$

That is, $D=398$ is such a strong Lochamp that it cannot be beaten for $D<1.5 \times$ $10^{5}$. In that respect, it is similar to the imaginary quadratic field with the notorious $D=-163$; see $[4$, Table 3].

5. Acknowledgements. The authors would like to thank both Pierre Barrucand and Daniel Shanks for their suggestions concerning this work.

Department of Computer Science

University of Manitoba

Winnipeg, Manitoba, Canada R3T 2N2

1. PIERRE A. BARRUCAND, "Sur certaines séries de Dirichlet," C. R. Acad. Sci Paris Sér. $A-B$, v. 269, 1969, A294-A296. MR 40 \#101.

2. M. D. HENDY, “The distribution of ideal class numbers of real quadratic fields," Math. Comp., v. 29, 1975, pp. 1129-1134.

3. E. L. INCE, "Cycles of reduced ideals in quadratic fields," Mathematical Tables, vol. IV, British Association for the Advancement of Science, London, 1934.

4. DANIEL SHANKS, Systematic Examination of Littlewood's Bounds on $L(1, x)$, Proc. Sympos. Pure Math., vol. 24, Amer. Math. Soc., Providence, R.I., 1973, pp. 267-283. MR 49 \# 2596.

5. H. J. S. SMITH, "Note on the theory of the Pellian equation and of binary quadratic forms of a positive discriminant," Proc. London Math. Soc., v. 7, 1875/76, pp. 199-208. 